\title{
The overheating effects in germanium quantum well with two subbands occupied
}

\author{
I.B. Berkutov ${ }^{1,2,3}$, V.V. Andrievskii ${ }^{1,2}$, Yu.A. Kolesnichenko ${ }^{1}$, Yu.F. Komnik ${ }^{1}$, \\ and O.A. Mironov ${ }^{2,4}$ \\ ${ }^{1}$ B. Verkin Institute for Low Temperature Physics and Engineering of the National Academy of Sciences of Ukraine \\ 47 Nauky Ave., Kharkiv 61103, Ukraine \\ E-mail: andrievskii@ilt.kharkov.ua \\ ${ }^{2}$ Institute of Low Temperature and Structure Research, Polish Academy of Sciences \\ ul. Okólna 2, 50-422, Wrocław, Poland \\ ${ }^{3}$ Department of Physics, North Carolina State University, Raleigh, NC 27695, USA \\ ${ }^{4}$ Department of Physics, University of Warwick, Coventry CV4 7AL, UK
}

Received March 29, 2018, published online June 27, 2018

\begin{abstract}
The charge carrier overheating effect was studied in the $p$-type $\mathrm{Si}_{0.4} \mathrm{Ge}_{0.6} / \mathrm{Ge} / \mathrm{Si}_{0.4} \mathrm{Ge}_{0.6}$ heterostructure with two subband occupy. The temperature dependences of hole-phonon relaxation time $\tau_{h \text {-ph }}$ at weak magnetic fields demonstrated transition of the 2D system from regime of "partial inelasticity" characterized by dependence $\tau_{h \text {-ph }}^{-1} \propto T^{2}$ to regime of small-angle scattering, described by dependence $\tau_{h \text {-ph }}^{-1} \propto T^{5}$ with temperature increase. But in higher magnetic fields the dependence $\tau_{h \text {-ph }}^{-1} \propto T^{3}$ manifests itself on dependences $\tau_{h \text {-ph }}\left(T_{h-\mathrm{ph}}\right)$. The possible explanations of such dependences are discussed.

PACS: 72.15.Lh Relaxation times and mean free path;

72.20.My Galvanomagnetic and other magnetotransport effects;

72.20.-i Conductivity phenomena in semiconductors and insulators.
\end{abstract}

Keywords: quantum well, overheating effects, acoustical coupling, the heat balance equation.

\section{Introduction}

One of the most important characteristics of charge carriers with two-dimensional spectrum in quantum wells, heterostructures, etc. is electron-phonon relaxation time $\tau_{e \text {-ph }}$ (or hole-phonon relaxation time for hole spectrum $\left.\tau_{h \text {-ph }}\right)$. It characterizes the excess energy which transvers from the electron to the phonon system under the influence of a high electric field (current) or of other, "heating", factors. It is advisable to use the electron overheating effect to determine this time. In the electron overheating effect the electron temperature $T_{e}$ exceeds the phonon temperature $T_{\mathrm{ph}}$ (which stays unchanged) under the influence of a high electric field. To realize the electron overheating effect it is necessary to ensure the free escape of phonons from the conducting layer into the surrounding crystal. The dielectric substrate with good acoustical coupling between metal and dielectric must be used when we deal with thin metal films, but this requirement is manifestly satisfied for quantum wells, because their surrounding crystal has the same acoustic properties as the material in which the quantum well is realized.

The transfer of the excess energy between electrons and crystal even in the condition of strong elastic scattering of electrons by impurities and lattice defects is controlled by $\tau_{e \text {-ph }}$. To determine time $\tau_{e \text {-ph }}$, the value of the temperature of overheated electrons in conditions of strong current flow $\Delta T_{e}=T_{e}-T_{\mathrm{ph}}$ must be found. The temperature dependence of the quantum corrections to the conductivity associated with the effects of weak localization and electron-electron interaction were using to determine electron temperature $T_{e}$ in number of earlier studies. This method of the $T_{e}$ determination was realized in relatively simple objects with two-dimensional electron spectrum such as thin metal films with size quantization [1] and in $\delta$ layers in the semiconductor crystal [2]. The temperature dependence of the Shubnikov-de Haas (SdH) oscillation amplitude can be used as a "thermometer" in more advanced conducting systems, such as inversion layers and quantum wells [3-6]. This method was used in this study. 


\section{Experimental technique and results obtained}

The $p$-type $\mathrm{Si}_{0.4} \mathrm{Ge}_{0.6} / \mathrm{Ge} / \mathrm{Si}_{0.4} \mathrm{Ge}_{0.6}$ quantum well made of $19.6 \mathrm{~nm}$ "pure" germanium $(0.98 \pm 0.01 \%)$ was studied. The sample was prepared in the Nano Silicon group, Department of Physics, University of Warwick, UK. The structure of the sample is shown in Fig. 1. The hybrid-epitaxy technique was used to grow the layers: UHV-CVD for the $\mathrm{Si}_{0.4} \mathrm{Ge}_{0.6}$ strain-tuning buffer and low temperature SS-MBE for "Ge" channel [7]. Carriers were introduced by modulation boron doped $\mathrm{Si}_{0.4} \mathrm{Ge}_{0.6}$ layer placed below the quantum well. The sample had the conventional Hall bar geometry. The conducting region had a width of $0.05 \mathrm{~mm}$, and the distance between the potential contacts was $0.5 \mathrm{~mm}$. The measurements were carried out at temperatures 1.5-100 K and magnetic fields up to $14 \mathrm{~T}$.

The well pronounced ( $\mathrm{SdH}$ ) oscillations (see Fig. 2) are presented on magnetic field dependences of resistance at low temperatures $(<10 \mathrm{~K})$. The relatively regular oscillations in low magnetic field (below $\sim 5.5 \mathrm{~K}$ ) change its shape with increasing magnetic field and demonstrate spin (Zeeman) splitting of Landau levels. In this paper we will concentrate on magnetic fields below $\sim 5.5 \mathrm{~T}$. This interval of magnetic fields under study can be divided in two regions: the region of classically low magnetic fields (lower $2 \mathrm{~T}$ ), where SdH oscillations with quantum numbers $v \geq 34$ are observed, and the region of higher magnetic fields $(v<34)$. The analysis of the magnetoresistance data in low magnetic fields (region I in Fig. 3(a)) which was performed using Fast Fourier Transformation (FFT) reviled just one frequency maximum (see Fig. 3(b)). Two frequency maximums (see Fig. 3(c)) were found in Fourier spectrum in higher magnetic fields (region II in Fig. 3(a)). Also, in these magnetic fields the weak $\mathrm{ShH}$ oscillations beats are observed and it can be evidence that there are two subbands occupied in the structure under study. In this case the investigation of the electron overheating effect can lead to manifestation of new peculiarities.

\begin{tabular}{|c|}
\hline $2 \mathrm{~nm} \mathrm{Si}$ \\
\hline $20 \mathrm{~nm} \mathrm{Si}_{0.4} \mathrm{Ge}_{0.6}$ \\
\hline $16 \mathrm{~nm} \mathrm{Ge}$ channel \\
\hline $15 \mathrm{~nm} \mathrm{Si}_{0.4} \mathrm{Ge}_{0.6}$ \\
\hline $5 \mathrm{~nm} \mathrm{Si}_{0.4} \mathrm{Ge}_{0.6}$ \\
$\mathrm{~B} @ 2 \cdot 10^{18} \mathrm{~cm}^{-2}$ \\
\hline $20 \mathrm{~nm} \mathrm{Si}_{0.4} \mathrm{Ge}_{0.6}$ \\
\hline $200 \mathrm{~nm} \mathrm{Si}_{0.4} \mathrm{Ge}_{0.6}$ \\
$\left(T_{G}=800{ }^{\circ} \mathrm{C}\right)$ \\
\hline $7 \mathrm{~mm} \mathrm{Si}_{0.4} \mathrm{Ge}_{0.6}$ graded buffer \\
\hline
\end{tabular}

Fig. 1. The sample structure.

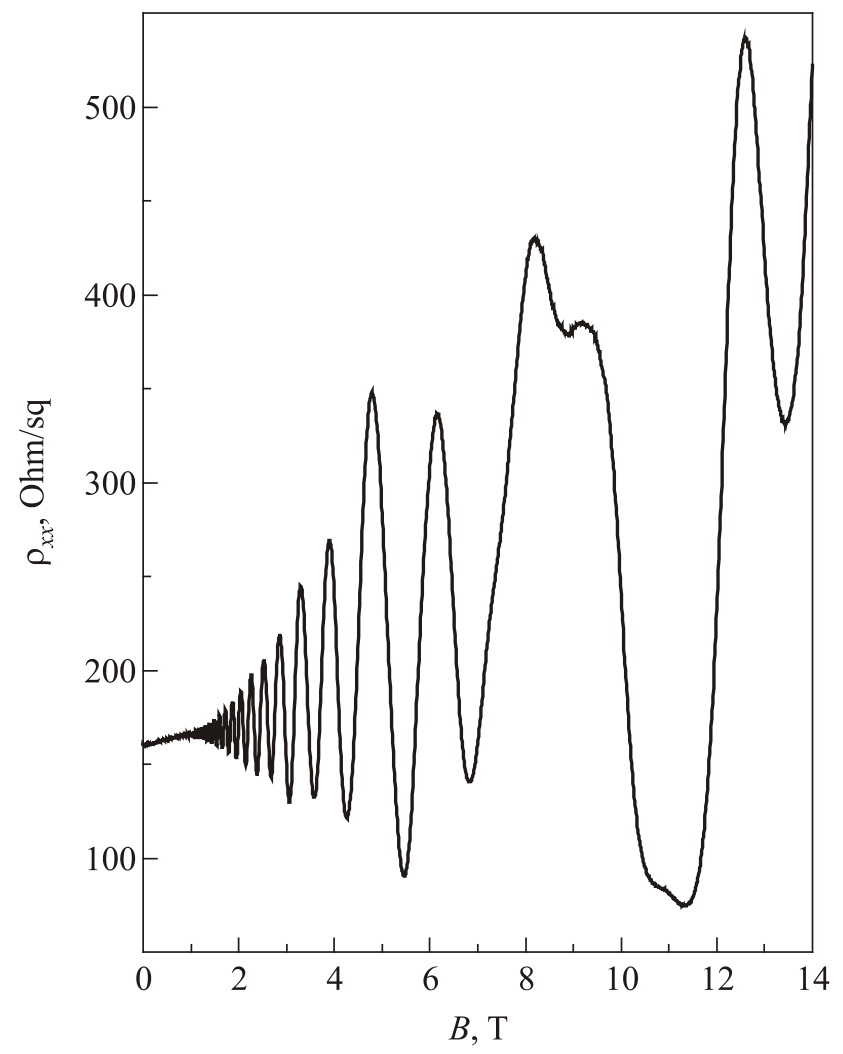

Fig. 2. The magnetic field dependence of sample resistance $\rho_{X X}(B)$ at temperature $T=1.45 \mathrm{~K}$.

The amplitudes of the SdH oscillations decrease with increasing of temperature and driving current (Fig. 4). The temperature of charge carriers (holes in our case) under conditions of high current can be determined from a comparison of the change in the amplitude of the SdH oscillations under the influence of electric current and under the influence of temperature [3,5]. Those dependences are shown in Fig. 5.
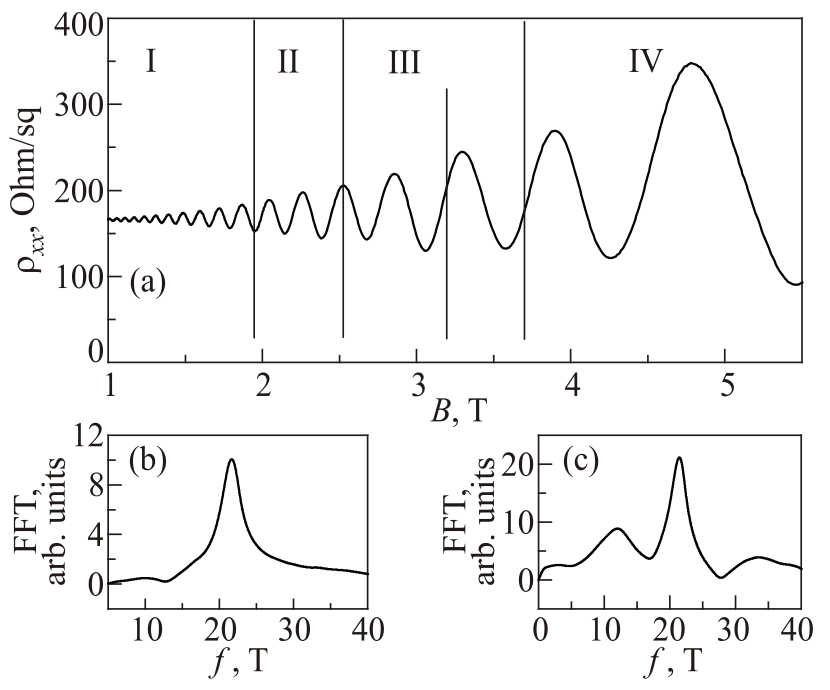

Fig. 3. The magnetic field dependence of sample resistance $\rho_{X X}(B)$ (a). FFT spectrum of SdH oscillations in magnetic fields from 1.2 to $3.2 \mathrm{~T}$ (b) and 1.2 to $5.5 \mathrm{~T}$ (c). 

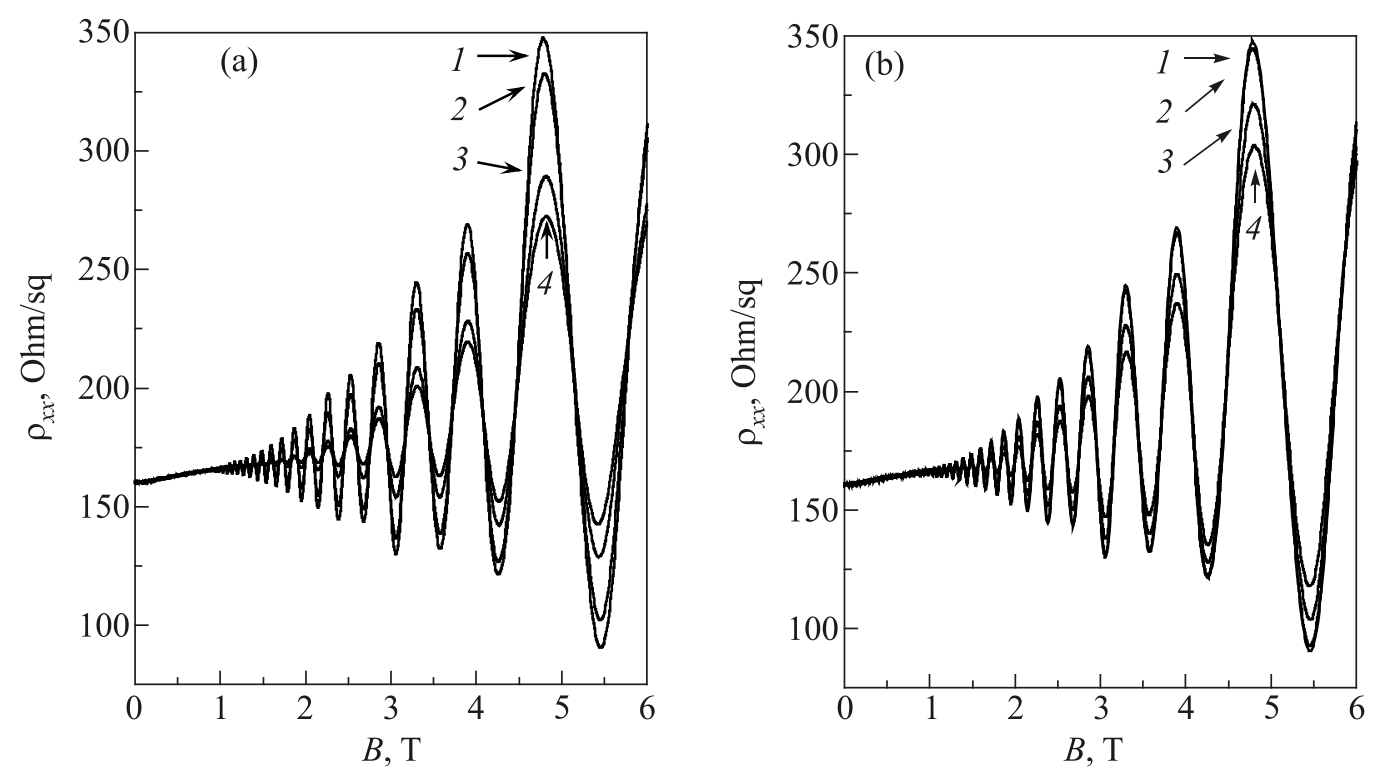

Fig. 4. The magnetic field dependences of sample resistance $\rho_{X X}(B)$ at temperatures (a): 1.45 (1), 2.5 (2), 4.5 (3), 5.5 (4) K, and currents (b): $100 \mathrm{nA}(1), 1 \mu \mathrm{A}(2), 5 \mu \mathrm{A}(3), 10 \mu \mathrm{A}$ (4).

The value of the electron-phonon relaxation time $\tau_{e \text {-ph }}$ can be calculated from the heat balance equation, where the supplied electrical power $P=E^{2} \sigma$ per unit volume is balanced with the amount of energy transferred by the electrons (holes) to the lattice per unit time:

$$
E^{2} \sigma=\int_{T_{\mathrm{ph}}}^{T_{e}} \frac{C_{e}(T) d T}{\tau_{e-\mathrm{ph}}(T)},
$$

here $C_{e}(T)$ is the heat capacity of electrons, $E$ is the electric field, $\sigma$ is the conductivity. If we assume, that in stationary state time $\tau_{e \text {-ph }}$ is corresponds to the temperature
$T_{e \text {-ph }}$, which characterizes electron-phonon interaction under electron overheating condition, and $C_{e}(T)=\gamma T$, then Eq. (1) changes to

$$
E^{2} \sigma=\left(T_{e}-T_{\mathrm{ph}}\right) \frac{T_{e}+T_{\mathrm{ph}}}{2} \frac{\gamma}{\tau_{e-\mathrm{ph}}\left(T_{e-\mathrm{ph}}\right)} .
$$

For calculation of $\tau_{e \text {-ph }}\left(T_{e \text {-ph }}\right)$ the Eq. (2) can be rewrites as [8]

$$
\left(T_{e}-T_{\mathrm{ph}}\right)=\frac{E^{2} \sigma}{\gamma T_{e-\mathrm{ph}}} \tau_{e-\mathrm{ph}}\left(T_{e-\mathrm{ph}}\right)
$$
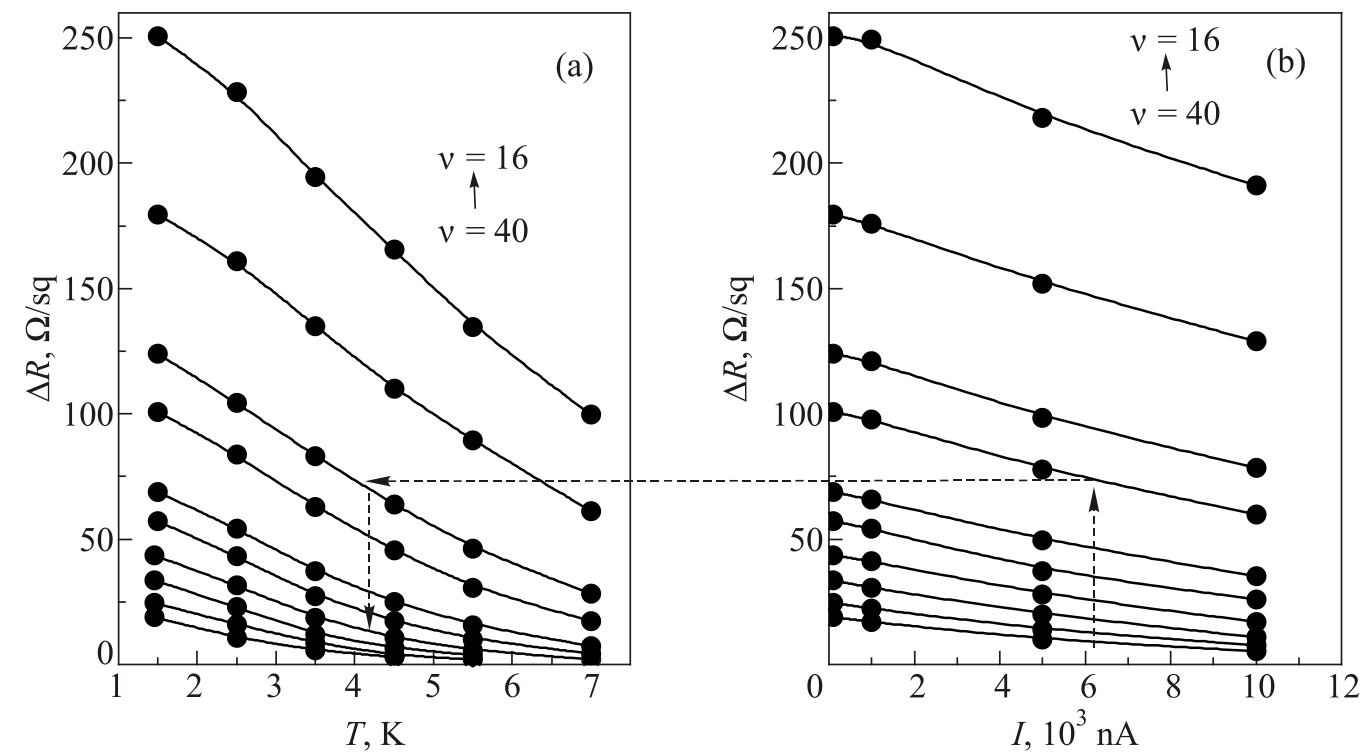

Fig. 5. Change in the amplitude of the SdH oscillations with quantum numbers $v=40(B=1.654 \mathrm{~T}), v=37(B=1.789 \mathrm{~T}), v=34$ $(B=1.951 \mathrm{~T}), v=31(B=2.145 \mathrm{~T}), v=28(B=2.383 \mathrm{~T}), v=25(B=2.678 \mathrm{~T}), v=22(B=3.054 \mathrm{~T}), v=19(B=3.576 \mathrm{~T}), v=16$ $(B=4.258 \mathrm{~T}), v=12(B=5.46 \mathrm{~T})$ upon changes in temperature (a) and current (b). 
here $T_{e-\mathrm{ph}}=1 / 2\left(T_{e}+T_{\mathrm{ph}}\right)$. Since the value of $\gamma$ is unknown for our object of study, we turn to the relation [9]

$$
\left(k T_{e}\right)^{2}-\left(k T_{\mathrm{ph}}\right)^{2}=\frac{6}{\pi^{2}}(e E)^{2} D \tau_{e-\mathrm{ph}},
$$

where $D$ is the diffusion coefficient. This relation is obtained from Eq. (3) with the electronic heat capacity and conductivity expressed in terms of the density of states $v_{d s}$ :

$$
C_{e}=\left(\frac{2}{3} \pi\right) k^{2} v_{d s} T \text { and } \sigma=e^{2} v_{d s} D
$$

For two-dimensional systems of charge carriers: $v_{d s}=m^{*} / \pi \hbar^{2}, D=1 / 2\left(v_{F}^{2} \tau\right)$, where $v_{F}=\left(\hbar / m^{*}\right)(2 \pi n)^{1 / 2}$ is a Fermi velocity, $n$ is the density of charge carriers, $\tau$ is the elastic scattering time, which determines the resistance per square $R_{\square}=m^{*} /\left(n e^{2} \tau\right)$ of the object under study ( $m^{*}$ is the effective mass). The electric field in a conducting channel of length $L$ and width $a$ can be found from the values of the current $I$ and the resistance per square $E=I R / L=I R_{\square} / a$.

The kinetic properties calculations of system under study were performed in Ref. 10. It was found that the values of effective mass, density and mobility of charge carriers are different on each subband. Note, according to calculations [10] the intersubband interaction is very weak, so each subband participates on a heat exchange process independently. The following values of kinetic characteristics were obtained: $m_{1}^{*}=0.112 m_{0}$ and $m_{2}^{*}=0.131 m_{0}$ on a first and second subbands, respectively $\left(m_{0}\right.$ is the free electron mass); $p_{1}=1.06 \cdot 10^{12} \mathrm{~cm}^{-2}$ and $p_{2}=0.61 \cdot 10^{12} \mathrm{~cm}^{-2}$; $\mu_{1}=2.66 \cdot 10^{4} \mathrm{~cm}^{2} /(\mathrm{V} \cdot \mathrm{s})$ and $\mu_{2}=1.84 \cdot 10^{4} \mathrm{~cm}^{2} /(\mathrm{V} \cdot \mathrm{s})$.

The characteristics obtained were used to calculate the diffusion coefficients of both subbands occupied. Then the heat balance equations, similar to Eq. (4), were composed for each subband, and temperature dependences of $\tau_{h \text {-ph }}$ were found. In Fig. 6 example of the temperature dependences of $\tau_{h \text {-ph }}\left(T_{h \text {-ph }}\right)$ for both subbands, which were calculated using $\mathrm{SdH}$ oscillations with filling factor $v=40$ is shown in bilogarithmical coordinates. The shape of the $\tau_{h \text {-ph }}\left(T_{h \text {-ph }}\right)$ dependence for upper subband is similar to this dependence for the lower subband, but it at several times smaller in magnitude, so we will concentrate on analysis of such dependences on lower subband. It was found that these dependences are different in various regions of magnetic fields. In first approximation it can be described by function $\tau_{h-\mathrm{ph}}^{-1} \sim T^{p}$. In low magnetic fields $(v \geq 40)$ and at low temperature $T_{h \text {-ph }}$ the dependence $\tau_{h \text {-ph }}^{-1} \sim T^{5}$ is observed (solid line on Fig. 7). Then it changes to dependence $\tau_{h \text {-ph }}^{-1} \sim T^{2}$ with temperature increasing (dash line in Fig. 7). The point of transition from dependence $\tau_{h \text {-ph }}^{-1} \sim T^{5}$ to $\tau_{h \text {-ph }}^{-1} \sim T^{2}$ shifts to lower temperatures with increasing of magnetic field (decreasing of $v$ ), and at $v=34$ dependence $\tau_{h \text {-ph }}^{-1} \sim T^{5}$ becomes almost unresolved. When mag-

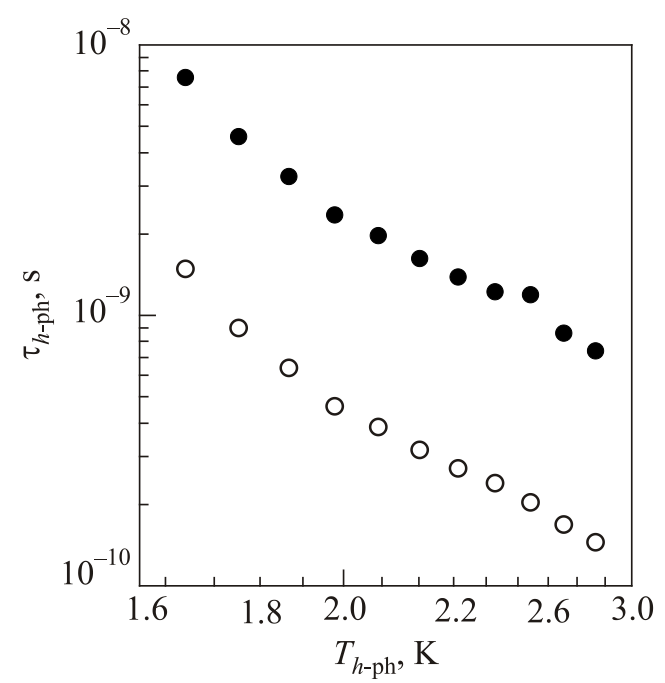

Fig. 6. Temperature dependence of hole-phonon relaxation times $\tau_{h \text {-ph }}$ calculated by Eq. (4) for the SdH oscillations with $v=40$. The empty symbols correspond to $\tau_{h \text {-ph }}$ values at upper subband and full symbols correspond to values at lower subband.

netic field increases further $(v<34)$, the dependence $\tau_{h \text {-ph }}^{-1} \sim T^{2}$ transfers to $\tau_{h-p h}^{-1} \sim T^{3}$, and from $v<22$ up to highest magnetic fields, only dependence $\tau_{h \text {-ph }}^{-1} \sim T^{3}$ is observed. Those changes in the shape of dependence $\tau_{h-\mathrm{ph}}^{-1} \sim T^{p}$ will be discussed in next section.

The study of the electron overheating effect in the structure with two subbands populated reviles one more feature. It was found that the amplitudes of the Fourier maximums with frequencies of $f_{1}$ and $f_{2}$ behave differently: with increasing of driving current the amplitude of the maximum $f_{1}$ decreases, but the amplitude of the maximum $f_{2}$ increases (Fig. 8). The position of the peaks on the frequency scale for all currents remains unchanged, which is indication that period SdH oscillations in inverse magnetic field stays constant and therefore density of carriers on both subbands occupied are unchanged.

The explanation of this feature is such that the electron overheating effect can manifest itself with different intensity at the group of charge carriers, which occupy different subbands. Using the values of charge carriers density and mobility, the resistances of the first $\left(\rho_{1}=221 \mathrm{Ohm}\right)$ and the second $\left(\rho_{2}=555 \mathrm{Ohm}\right)$ conducting channels were found. The experimental resistance measured in zero magnetic fields $\left(\rho_{0}=160 \mathrm{Ohm}\right)$ is close to the value $158.06 \mathrm{Ohm}$. The driving current which uses for the charge carriers heating distributes in two parallel circuits according to their conductivity. As a result, the electron overheating effect appears to be much weaker in the channel with low conductivity, which corresponds of frequency $f_{2}$. However, when current increases, the energy distribution between the subbands can be change under the influence of the electron-phonon interaction. 

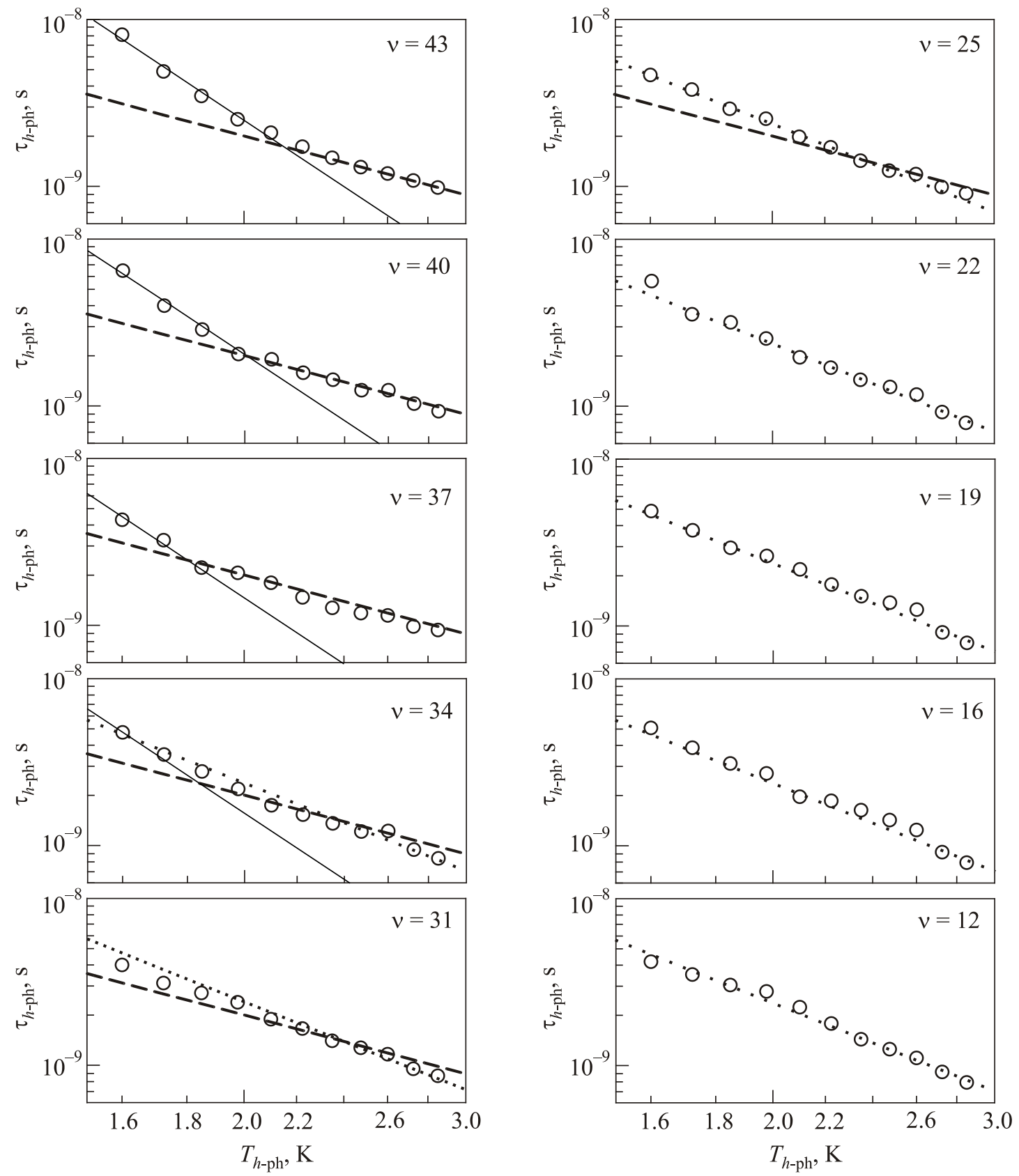

Fig. 7. Temperature dependences of hole-phonon relaxation times $\tau_{h \text {-ph }}$ calculated by Eq. (5) for the SdH oscillations with different quantum numbers. Solid line is the dependence $\tau_{h \text {-ph }}^{-1} \sim T^{5}$, dashed line is the dependence $\tau_{h \text {-ph }}^{-1} \sim T^{2}$, dotted line is the dependence $\tau_{h-\mathrm{ph}}^{-1} \sim T^{3}$.

\section{Discussion}

The conception which describes the electron-phonon relaxation in systems with two-dimensional spectrum was developed in theoretical studies [11-13]. In such systems the movement of electrons across the quantum well is quantized and phonons, which participate in electron-phonon interaction, are emitted or absorbed in direction predominantly perpendicular to the walls of the quantum well. In fact, the case of one subband was discussed in Refs. 11-13. At high temperature the wave vector of thermal phonons is $q_{T}=k_{B} T /(\hbar s)>2 \pi \hbar / \alpha$ ( $\alpha$ is the quantum well width, $s$ is the speed of sound), but it changes to $q_{T}<2 \pi \hbar / \alpha$ with temperature decreasing. The low-temperature case was observed in Ref. 11. In Refs. 12, 13 the region of temperatures, in which the wave momentum of a thermal phonon is sufficient to change the electron wave vector by the maximum value $2 k_{F}$, was described as region of "partial inelasticity". At lower temperatures, where $q_{T}<2 k_{F}$, only smallangle scattering of the electrons is possible. The scattering on deformation potential (DA) and piezoelectric scattering (PA) were observed in Refs. 11-13. The expressions for the inverse scattering time of electron-phonon interaction and the power of energy loss, when electrons and acoustic 

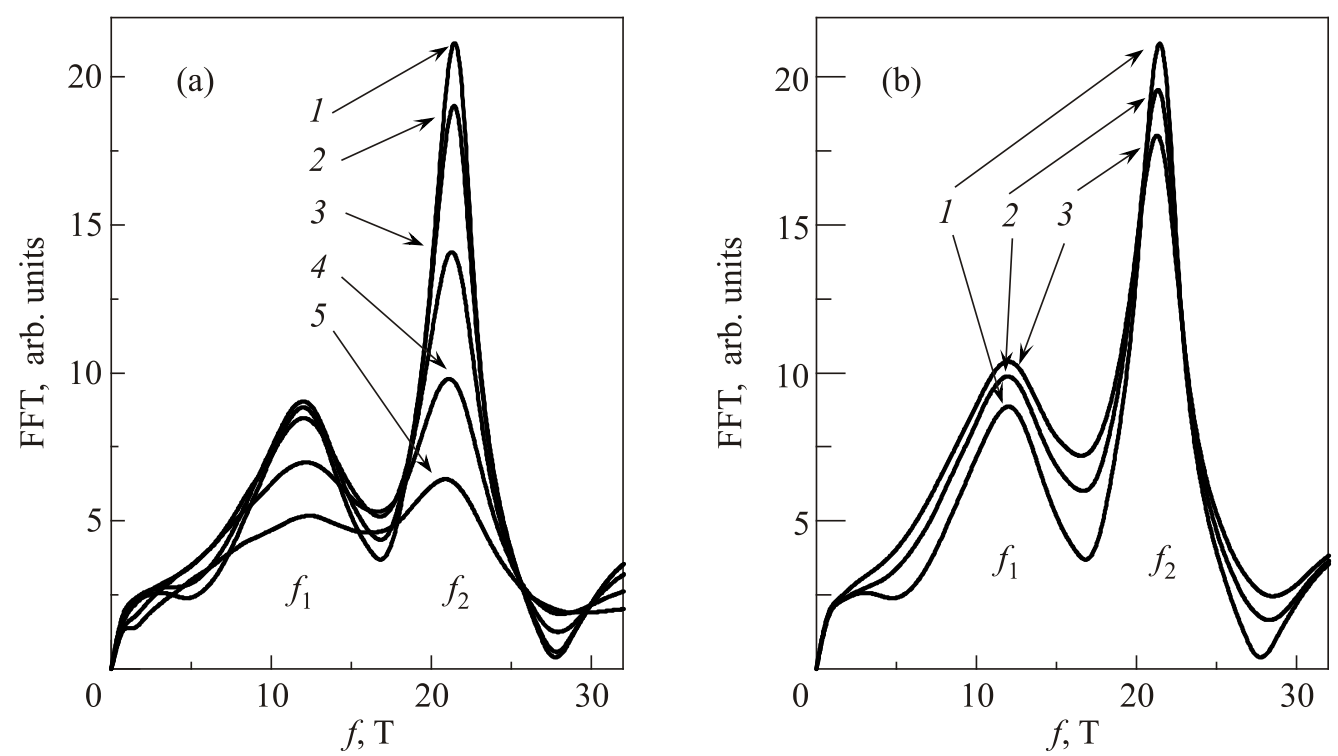

Fig. 8. FFT spectrum of SdH oscillations at temperatures (a): 1.45 (1), 2.5 (2), 4.5 (3), 7 (4), 10 (5) K, and currents (b): 100 nA (1), $5 \mu \mathrm{A}(2), 10 \mu \mathrm{A}(3)$.

phonons of the crystal lattice exchange their energy, were obtained. The equation for the power of energy loss can be derived from the heat balance Eq. (1). If we assume, that $\tau_{h-\mathrm{ph}}^{-1}=A T^{p}$ and $C_{e}=\gamma T$ the Eq. (1) can be changed as:

$$
E^{2} \sigma=\frac{A \gamma}{p+2}\left(T_{e}^{p+2}-T_{p h}^{p+2}\right) .
$$

Note, that the power of $T_{e}$ and $T_{\mathrm{ph}}$ two unities higher than power of $\tau_{e \text {-ph }}$ in Eq. (4). The powers $p$ for temperatures $T_{e}$ and $T_{\mathrm{ph}}$ in the energy loss equation equal 7 for DA scattering and 5 for PA scattering, as it shown in Ref. 11. According to Ref. 12 the dependence $\tau_{e \text {-ph }}^{-1} \propto T^{2}$ should be observed for DA in the region of "partial inelasticity" scattering, where $q_{T} \gg 2 k_{F}$, and dependence $\tau_{e \text {-ph }}^{-1} \propto T^{5}$ will be seen in the region of small angle scattering, where $q_{T}<2 k_{F}$. For PA scattering the dependence $\tau_{e-\mathrm{ph}}^{-1} \propto T$ will be observed in the region of partial "inelasticity", and dependence of $\tau_{e-\mathrm{ph}}^{-1} \propto T^{3}$ will be observed in the region of the small angle scattering [13]. The cross point from the partially inelastic scattering to small-angle scattering is determined by equation $q_{T}=2 k_{F}$. In the investigated case, this criterion does not correspond to the real situation, because it was formulated for one subband occupied case. When two subbands are occupied, the transition region can be diffused. Also, the theory [12,13] was developed in case of the absence of magnetic field, but the spectrum of phonons, which participate in electron-phonon interaction process, changes in the presence of magnetic field [14]. This refers to phonons, which propagate along the quantum well. In the absence of a magnetic field the relaxation of electrons is determined by the equally probable in the direction of emission of acoustic phonons, which propagate along the quantum well. When magnetic field is applied for phonons emitted along the well, the momentum is limited by the magnetic length and therefore the energy relaxation rate decreases and the electron-phonon relaxation time increases.

In our opinion the experimental results (Fig. 7) are related to the transition region from the regime of "partial" inelasticity to regime of small-angle scattering. In previous experiments, performed in SiGe quantum wells with one subband occupied [3-6], the regions with $\tau_{e-p h}^{-1} \propto T^{2}$ and $\tau_{e-\mathrm{ph}}^{-1} \propto T^{5}$ were clearly resolved on dependences $\tau_{h-\mathrm{ph}}\left(T_{h-\mathrm{ph}}\right)$, but the transition region was not discussed in those papers. For sample under study with two subbands occupied the transition region appears to be noticeable on the temperature scale. As it was noticed above, the regions with dependence $\tau_{e \text {-ph }}^{-1} \propto T^{5}$ at low temperatures and with dependence $\tau_{h \text {-ph }}^{-1} \propto T^{2}$ at higher temperatures are clearly resolved for $v=43,40$ and 37 on dependences $\tau_{h-\mathrm{ph}}\left(T_{h \text {-ph }}\right)$ in Fig. 7. But in higher magnetic fields $(v \leq 31)$ the dependence $\tau_{h \text {-ph }}^{-1} \propto T^{3}$ manifests itself on dependences $\tau_{h \text {-ph }}\left(T_{h \text {-ph }}\right)$. We assume, that their appearance is connected to mixing of two kinds of dependences with $T^{2}$ and $T^{5}$. An important conclusion follows from these observations: In a weak magnetic field small angle processes of electron-phonon scattering appear in about the same temperature range as in the absence of a magnetic field, but it shifts to lower magnetic fields with magnetic field increasing, and only "partially" inelastic processes of electron-phonon scattering (as it named in Ref. 12) are exhibit itself in high magnetic fields. Note, that processes of small angle scattering are observed in region I in Fig. 3(a), in which only one peak on Fourier spectrum is present, and it means that the analysis presented above describes processes associated only with lower subband. In this range of magnetic fields, the degeneracy of the upper subband does not appear because of effect the Landau levels thermal broadening. When magnetic field increases (region II in Fig. 3(a)) the Landau le- 
vels of the second subband become clearly resolved leading to the observation of common contribution to the electron-phonon interaction from both subbands. The dependence $\tau_{h-p h}^{-1} \propto T^{3}$ apparently is mixture state of two components: the processes of "partial inelasticity" with $\tau_{h \text {-ph }}^{-1} \propto T^{2}$ on the lower subband and the small-angle scattering processes with $\tau_{h-\mathrm{nh}}^{-1} \propto T^{5}$ on the upper subband.

Another possible explanation of observation of $\tau_{h-p h}^{-1} \propto T^{3}$ dependence is the assumption of a dominant role of the piezoelectric scattering in strong magnetic field. However, the value of the piezoelectric interaction constant $e_{p z}=9.6 \cdot 10^{-2} \mathrm{C} / \mathrm{m}^{-2}$, obtained by analysis of experimental data, which was perfumed in the same way as it was done in Ref. 15, appears to be significantly higher than the value $e_{p z}=3.4 \cdot 10^{-3} \mathrm{C} / \mathrm{m}^{-2}$ obtained in that paper. The contribution of the piezoelectric scattering may be presented in the formation of the temperature dependence, but it is not determinative, as it follows from theoretical calculations, performed for semiconductor crystals, which were not included in the category of piezoelectric materials [11].

\section{Conclusion}

Thus, the study of the electron overheating effect in germanium quantum well with two subbands occupied, performed in order to obtain information about the temperature dependence of the electron-phonon scattering revealed new features of the charge carriers scattering in such object: i) It was demonstrated, that in two-dimensional conduction system with two subbands occupied the electron transport is performed by two groups of charge carriers, which act in parallel and independently (when interaction between these groups is weak). ii) It was found, that the temperature dependence of the electron-phonon scattering in the temperature range $T_{h \text {-ph }}=1.5-3 \mathrm{~K}$ described by function $\tau_{h-\mathrm{ph}}^{-1} \propto T^{p}$. In weak magnetic fields the dependence $\tau_{h \text {-ph }}(T)$, where $p=5$, manifests itself at lowest temperatures, but at higher temperatures it turns to dependence with $p=2$. In strong magnetic fields typical dependence is $p=3$, which was interpreted as the result of mixing of partially inelastic scattering processes, characterized by dependence $\tau_{h \text {-ph }}^{-1} \propto T^{2}$ and small-angle scattering processes, described by dependence $\tau_{h \text {-ph }}^{-1} \propto T^{5}$. iii) It was found, that the magnetic field affects not only to the contributions of these two types of scattering mechanisms to the temperature dependence $T_{h \text {-ph }}$, but also on the contributions from two groups of carriers from different subbands, for which the processes of carrier heating happen not simultaneously.

1. V.Yu. Kashirin and Yu.F. Komnik, Fiz. Nizk. Temp. 19, 410 (1993) [Low Temp. Phys. 19, 288 (1993)].

2. V.Yu. Kashirin, Yu.F. Komnik, O.A. Mironov, C.J. Emelius, and T.E. Whall, Fiz. Nizk. Temp. 22, 1174 (1996) [Low Temp. Phys. 22, 642 (1996)].
3. V.V. Andrievskii, I.B. Berkutov, Yu.F. Komnik, O.A. Mironov, and T.E. Whall, Fiz. Nizk. Temp. 26, 1202 (2000) [Low Temp. Phys. 26, 890 (2000)].

4. I.B. Berkutov, V.V. Andrievskii, Yu.F. Komnik, M. Myronov, and O.A. Mironov, Fiz. Nizk. Temp. 34, 1192 (2008) [Low Temp. Phys. 34, 943 (2008)].

5. I.B. Berkutov, V.V. Andrievskii, Yu.F. Komnik, and O.A. Mironov, Materialwissenschaft und Werkstofftechnik 42, 15 (2011).

6. I.B. Berkutov, Acta Physica Polonica A 119, 228 (2011).

7. R.J.H. Morris, T.J. Grasby, R. Hammond, M. Myronov, O.A. Mironov, D.R. Leadley, T.E. Whall, E.H.C. Parker, M.T. Currie, C.W. Leitz, and E.A. Fitzgerald, Semicond. Sci. Technol. 19, L106 (2004).

8. P.W. Anderson, E. Abrahams, and T.V. Ramakrishnan, Phys. Rev. Lett. 43, 718 (1979).

9. S. Hershfield and V. Ambegaokar, Phys. Rev. B 34, 2147 (1986).

10. I.B. Berkutov, V.V. Andrievskii, Yu. Komnik, and O.A. Mironov, Fiz. Nizk. Temp. 43, 1515 (2017) [Low Temp. Phys. 43, 1208 (2017)].

11. P.J. Prise, J. Appl. Phys. 53, 6863 (1982).

12. V. Karpus, Fiz. Tekh. Poluprovodn. 20, 12 (1986) [Sov. Phys. Semicond. 20, 6 (1986)].

13. V. Karpus, Fiz. Tekh. Poluprovodn. 21, 1949 (1987) [Sov. Phys. Semicond. 21, 1180 (1987)].

14. G.N. Gol'tsman and K.V. Smirnov, Pis'ma Zh. Eksp. Teor. Fiz. 74, 532 (2001) [JETP Lett. 74, 474 (2001)].

15. R. Leturcq, D. L’Höte, R. Tourbot, V. Senz, U. Gennser, T. Ihn, K. Ensslin, G. Dehlinger, and D. Grützmacher, Europhys. Lett. 61 (4), 499 (2003).

\section{Ефекти перегріву в квантовій ямі германію з двома зайнятими підзонами}

\section{І.Б. Беркутов, В.В. Андрієвський, Ю.О. Колесниченко, Ю.Ф. Комнік, О.А. Міронов}

Ефект перегріву носіїв заряду вивчався в гетероструктурі $\mathrm{Si}_{0,4} \mathrm{Ge}_{0,6} / \mathrm{Ge} / \mathrm{Si}_{0,4} \mathrm{Ge}_{0,6}$ р-типу $з$ двома зайнятими підзонами. В слабких магнітних полях температурні залежності часу дірково-фононної релаксації $\tau_{h \text {-ph }}$ демонструють перехід двовимірної системи $з$ режиму «часткової непружності», яка характеризується залежністю $\tau_{h-\mathrm{ph}}^{-1} \propto T^{2}$, до режиму малокутового розсіювання, що описується залежністю $\tau_{h \text {-ph }}^{-1} \propto T^{5}$ з підвищенням температури. В більш високих магнітних полях залежність $\tau_{h \text {-ph }}^{-1} \propto T^{3}$ змінюється на $\tau_{h \text {-ph }}\left(T_{h \text {-ph }}\right)$. Обговорюються можливі варіанти пояснення таких спостережень.

Ключові слова: квантова яма, ефекти перегріву, акустичне з'єднання, рівняння теплового балансу. 